\title{
Immediate neurophysiological effects of transcranial electrical stimulation
}

\author{
Anli Liu (10) 1,2, Mihály Vöröslakos ${ }^{3,4}$, Greg Kronberg ${ }^{5}$, Simon Henin (10) 1,2, \\ Matthew R. Krause (10 ${ }^{6}$, Yu Huang ${ }^{5}$, Alexander Opitz ${ }^{7}$, Ashesh Mehta ${ }^{8,9}$, \\ Christopher C. Pack ${ }^{6}$, Bart Krekelberg ${ }^{10}$, Antal Berényi (i) ${ }^{3}$, Lucas C. Parra (i) ${ }^{5}$, \\ Lucia Melloni ${ }^{1,2,11}$, Orrin Devinsky ${ }^{1,2}$ \& György Buzsáki (i) ${ }^{4}$
}

Noninvasive brain stimulation techniques are used in experimental and clinical fields for their potential effects on brain network dynamics and behavior. Transcranial electrical stimulation (TES), including transcranial direct current stimulation (tDCS) and transcranial alternating current stimulation (tACS), has gained popularity because of its convenience and potential as a chronic therapy. However, a mechanistic understanding of TES has lagged behind its widespread adoption. Here, we review data and modelling on the immediate neurophysiological effects of TES in vitro as well as in vivo in both humans and other animals. While it remains unclear how typical TES protocols affect neural activity, we propose that validated models of current flow should inform study design and artifacts should be carefully excluded during signal recording and analysis. Potential indirect effects of TES (e.g., peripheral stimulation) should be investigated in more detail and further explored in experimental designs. We also consider how novel technologies may stimulate the next generation of TES experiments and devices, thus enhancing validity, specificity, and reproducibility.

- lectrical stimulation to the brain has a long history in both science and medicine. In 1867, Helmholtz discussed how electrical currents applied to the head can generate visual sensations ${ }^{1}$. Currents as low as $0.3 \mathrm{~mA}$ could induce phosphenes, and stronger currents could induce brighter and more lasting visual effects ${ }^{2}$. Subsequent studies confirmed that these visual phenomena result from retinal rather than direct brain stimulation ${ }^{3,4}$.

Early noninvasive electrical stimulation technologies used high intensities to directly affect brain activity (see Box 1). Electroconvulsive therapy, introduced into psychiatry in the 1930s, used currents of up to $60 \mathrm{~mA}$ to induce generalized seizures ${ }^{5}$. Subsequent studies on

\footnotetext{
${ }^{1}$ New York University Comprehensive Epilepsy Center, 223 34th Street, New York, NY 10016, USA. ${ }^{2}$ Department of Neurology, NYU Langone Health, 222 East 41st Street, 14th Floor, New York, NY 10016, USA. ${ }^{3}$ MTA-SZTE 'Momentum' Oscillatory Neuronal Networks Research Group, Department of Physiology, Faculty of Medicine, University of Szeged, 10 Dom sq., Szeged H-6720, Hungary. ${ }^{4}$ New York University Neuroscience Institute, 435 East 30 th Street, New York, NY 10016, USA. ${ }^{5}$ Department of Biomedical Engineering, City College of New York, 160 Convent Ave, New York, NY 10031 , USA. ${ }^{6}$ Montreal Neurological Institute, McGill University, Montreal, QC H3A 2B4, Canada. ${ }^{7}$ Department of Biomedical Engineering of Minnesota, 312 Church St. SE, Minneapolis, MN 55455, USA. ${ }^{8}$ Department of Neurosurgery, Hofstra Northwell School of Medicine, 611 Northern Blvd, Great Neck, NY 11021 , USA. ${ }^{9}$ Feinstein Institute for Medical Research, Hofstra Northwell School of Medicine, 350 Community Drive, Manhasset, NY 11030 , USA. ${ }^{10}$ Center for Molecular and Behavioral Neuroscience, Rutgers University, 197 University Avenue, Newark, NJ 07102, USA. ${ }^{11}$ Max Planck Institute for Empirical Aesthetics, Grüneburgweg 14, 60322 Frankfurt am Main, Germany. Correspondence and requests for materials should be addressed to A.L. (email: anli.liu@nyumc.org) or to G.Bák. (email: gyorgy.buzsaki@nyumc.org)
} 


\section{Box 1 Terminology}

Transcranial electrical stimulation (TES) - The technique of applying an electric field at the scalp surface with the purpose of directly affecting brain activity. Early efforts applied fields at high intensities for electroconvulsive therapy (ECT, $60 \mathrm{~mA}$ ), electroanesthesia (40 mA), and electrosleep (3-10 mA). More recent efforts have applied TES at lower intensities (typically 1-2 mA) to reduce peripheral side effects, such as skin sensation and phosphenes. Direct current stimulation-Electrical current applied in a constant, unidirectional manner flowing from anode to cathode. When DC stimulation is applied across the scalp, it is commonly referred to as transcranial direct current stimulation (TDCS).

Alternating current stimulation-Electrical current applied in a varying, typically sinusoidal, waveform, with current flowing from anode to cathode in one half-cycle and in the reverse direction in the second half-cycle. When the AC stimulation is applied across the scalp, it is commonly referred to as transcranial alternating current stimulation (TACS).

Electric field-The difference in voltage between two locations (in the brain). The electric field is a vector with both magnitude and direction, and is measured in units of Volts/meter $(\mathrm{V} / \mathrm{m})$.

Direct effects-Potential effects of TES on the excitability of neurons in the brain, which include both immediate and cumulative effects.

Indirect effects-Potential side effects of TES on non-neuronal elements, including placebo, peripheral nerves, retina, cochlea, glia, immune system, and blood flow as well as cumulative, long-term metabolic effects on neurons.

Immediate effects-Potential effects of TES which occur simultaneously or acutely during stimulation. These effects are measured by changes in neuronal firing patterns or the local field potential (defined below). We use the terms acute, concurrent, or online as synonyms of the term "immediate." Offline effects-Potential effects of TES which may outlast the period of stimulation.

Local field potential-a composite measure of electric current generated from all active cellular processes within a volume of brain tissue superimpose at a given location in the extracellular medium and generate an extracellular potential $V_{\mathrm{e}}$ (a scalar measured in Volts) with respect to a reference potential. The difference in $V_{e}$ between two locations gives rise to an electric field (a vector, $V / m$ ). The local field is monitored by small intracranial electrodes as opposed to those obtained by scalp EEG recordings.

Oscillations-Rhythmic fluctuations in the local field potential, which can range in frequency from ultraslow $(0.05 \mathrm{~Hz})$ to ultrafast $(500 \mathrm{~Hz})$, with specific oscillation frequencies characterizing particular brain states.

Shunting-During TES application, the flow of current away from the brain due to passage along a low-resistance pathway (skin, subcutaneous tissue), and away from a high resistance pathway (the skull). Current shunting explains why only a small part of the current delivered through TES electrodes actually reaches the brain surface.

electroanesthesia and electrosleep used subconvulsive current intensities, delivered through large copper or platinum disks covered with saline-soaked gauze over frontal and occipital scalp, to affect large neocortical regions. To induce anesthesia, up to $40 \mathrm{~mA}$ direct currents (DC) or alternating currents (AC; 1 $\mathrm{Hz}-200 \mathrm{~Hz}$ ) were used (See Box 1), while weaker intensities (3-10 mA) were typically needed for sleep induction ${ }^{6}$. More than 500 human surgeries were carried out under electrical anesthesia supplemented with medication ${ }^{7}$, but serious side effects led to the decline of the technique.

The remaining present-day application of high-intensity transcranial electrical stimulation (TES, see Box 1) is for intraoperative neuromonitoring. This technique, introduced by Merton and Merton (1980), uses high-intensity stimulation (up to $2000 \mathrm{~V}$ ) through a pair of electrodes positioned over primary motor cortex to generate a visible twitch in the contralateral hand to monitor the functional integrity of central motor pathways during resective surgery ${ }^{8-10}$. These early applications for highintensity TES $6,11,12$ laid the groundwork for subsequent experiments which suggested that weak currents applied to the scalp can also induce behavioral effects but without side effects and without conscious awareness of the stimulation ${ }^{13,14}$. Compared to other noninvasive neuromodulatory techniques such as transcranial magnetic stimulation (TMS) and ultrasound stimulation $^{15}$, advantages of TES include low cost, portability, and potential in-home applications, fueling a proliferation of human trials ${ }^{16,17}$. However, a disadvantage of TES is that it may activate excitable peripheral elements between the scalp electrodes, including trigeminal nerve branches, the greater occipital nerve, retina, and vestibular organs.

Despite more than 4000 publications (PubMed) on TES in the past decade, we lack a mechanistic understanding of the mechanism (or mechanisms) by which this technique produces beneficial or deleterious effects. Most TES studies place an electrode above a targeted cortical region with the assumption that the underlying neuronal activity will be boosted or suppressed. While experiments in intact animals and computational models have studied physiological and behavioral effects of TES, the mechanisms are incompletely understood and their translation to humans even more uncertain. It is also unclear whether TES works through direct or indirect effects (see Box 1). This mechanistic uncertainty explains why behavioral effects in humans are often weak, variable and difficult to replicate ${ }^{18,19}$.

In this Review, we discuss the immediate (i.e., acute, concurrent, or online, see Box 1) neurophysiological effects of electrical field stimulation in brain slices, rodents, non-human primates, and humans. We compare experimental findings with computational models, highlight the gaps in understanding, and discuss novel methods to deliver spatially precise stimulation at higher intensities than conventionally used, while reducing unwanted peripheral effects. We close with recommendations for future clinical and translational TES investigations. We do not discuss here potential offline effects of TES, i.e. those effects which occur or persist after the current has been switched off (see Box 1). These have been reviewed elsewhere ${ }^{20,21}$.

\section{Biophysics of induced electric fields}

At each point within the brain, a scalar electric potential can be measured relative to an arbitrary reference and expressed in Volts (V). The electric field $\vec{E}$ is the local change (gradient) of the voltage; $\vec{E}$ is a vector whose amplitude is measured in Volts per meter $(\mathrm{V} / \mathrm{m})$. All transmembrane currents from nearby neuronal processes contribute to generate $\vec{E} 22$.

When a current is applied to brain tissue (as in TES), it affects the polarization of cellular membranes, which in turn can alter neuronal excitability. Terzuolo and Bullock ${ }^{23}$ first demonstrated this using DC stimulation applied with metal bars to the crayfish abdominal receptor and lobster cardiac ganglion neurons. Induced electric fields as low as $1 \mathrm{~V} / \mathrm{m}$ affected the timing of action potentials while much higher fields triggered action potentials in silent neurons ${ }^{23}$. However, the orientation of the field was also important: rotating the applied electric field's orientation relative to the main neuron's soma-dendritic axis (e.g., from parallel to perpendicular to the main neuronal axis) affected the strength and direction of the effects. These findings 
generalize to the mammalian brain. An anode on the pial surface (cathode at depth) depolarizes neurons and increases neuronal firing frequency, while the reversed current flow (surface cathode, depth anode) hyperpolarizes neurons and reduces their firing rates $^{24,25}$.

Yet, the above picture is an oversimplification. First, it assumes that the soma is singularly affected, but axon initial segment, dendrites, and axon are also affected with different, unknown sensitivities to electric fields. These combined and variable effects on neuronal compartments can alter neuronal spiking ${ }^{19}$. In addition, tonic depolarization of axon terminals could release neurotransmitters in an axon branch-specific manner without generating action potentials. Second, although fields perpendicular to the soma-dendritic axis may have little influence on the apical dendrites of pyramidal neurons ${ }^{26-28}$, they can activate basal dendrites and dense local axonal arbors of basket and chandelier interneurons. In addition, neurons with star-shaped dendrites-many inhibitory interneurons, layer 4 stellate cells, thalamocortical cells, and basal ganglia neurons-might be affected equally by all field orientations. These complexities (Fig. 1) are relevant because the orientation of TES-induced intracranial fields relative to these different neuronal populations and compartments varies considerably ${ }^{29}$. In summary, the influence of TES current depends not only on the amplitude but upon how the induced electric field relates to the threedimensional organization of neuronal compartments ${ }^{28,30,31}$. Biophysical experiments and detailed computational models are needed to fully understand these complexities.

\section{Hypothesized immediate effects of electric fields on neural activity}

We define immediate effects of TES as those that occur simultaneously or acutely from the modulation of the membrane potential by the external field. For a sufficiently strong electric field, neuronal firing rates should result from changes in a neuron's input/output function ${ }^{19}$, facilitated neurotransmitter release from presynaptic terminals ${ }^{32}$, or ectopically induced spikes ${ }^{33}$.

With AC stimulation, current flows from anode to cathode in one half-cycle and reverses direction during the other half-cycle. These half-cycles fields are equal magnitude but opposite direction. Thus, during low-frequency AC stimulation, somata may be alternately depolarized and hyperpolarized, which could mimic effects of anodal and cathodal DC stimulation. If the applied AC frequency is much faster than the neuronal membrane time

\section{$\oplus \oplus \oplus \oplus \oplus \oplus \oplus \oplus \oplus \oplus \oplus \oplus \oplus \oplus \oplus \oplus \oplus$}

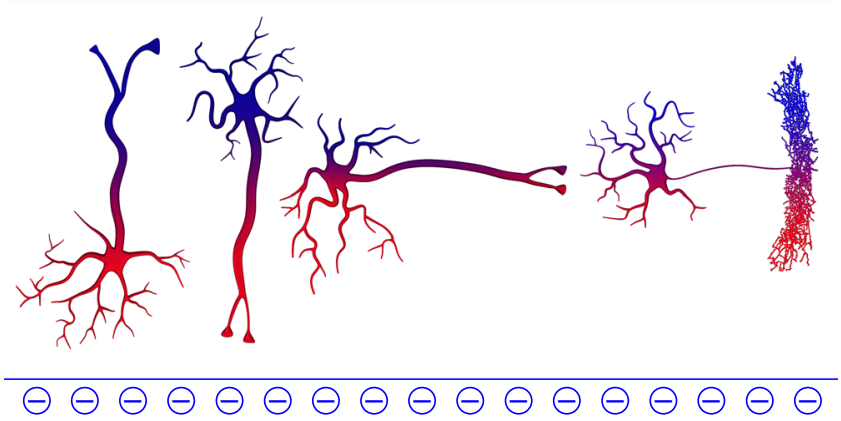

Fig. 1 The impact of orientation of neuronal compartments on TES-induced excitability. Four idealized neurons are shown with different orientation relative to the induced electric field. The electric field can affect the soma, dendrites, axon initial segment and the axon tree differently. The relationship between the electric field vector and the morphology/ orientation of neurons and individual neuronal compartments determine whether the neuron will be net depolarized or hyperpolarized constant $(\sim 30 \mathrm{~ms}$, or $>33.3 \mathrm{~Hz})$, the fast-changing polarities of the stimulation may reduce neuronal polarization ${ }^{34,35}$, and thereby reduce unwanted peripheral nerve stimulation effects (see below).

In tACS studies, applied TES current is posited to affect the brain's native oscillatory patterns ${ }^{36-38}$ and thereby target cognitive and therapeutic applications. While the magnitude of TESinduced physiological effects varies with the strength of the induced field, the 'effectiveness' of the stimulation and its consequences on neuronal and network activity depend on many other experimental variables and the brain's state. We distinguish five neural mechanisms that could affect network activity: stochastic resonance, rhythm resonance, temporal biasing of neuronal spikes, entrainment of network patterns, and imposed patterns (Fig. 2). These five mechanisms of field-induced effects can cooperate or compete with each other and with endogenous brain activity, and can occur simultaneously in different networks of the same brain.

(1) Stochastic resonance. Because physiological (EPSP, IPSP) and exogenous (TES) polarizing mechanisms are added, there is no theoretical 'minimum effective threshold' of the induced electric field ${ }^{39,40}$. When a neuron nears the threshold of spike generation, a very small amount of applied field can bias spike timing or spike probability. This is known as 'stochastic resonance' 41 . Weak stochastic effects are hard to quantify in normal networks with fluctuating activity levels because the affected neurons may be scattered in distant networks. Upon repeated application of the field, different neurons may be excited or hyperpolarized, and thus it may be difficult to generate reproducible effects based on this mechanism alone. (2) Rhythm resonance. Under well-controlled, closed-loop conditions, a very weak field can be precisely timed to the depolarizing phase of an oscillating neuron's membrane potential (see below). Even without a closed-loop system, rhythm resonance may occur when an AC field is applied to a regular endogenous rhythm at the same frequency, because the external field can affect the native oscillation at a similar phase during each cycle ${ }^{42-50}$. Such phase matching may explain tACS beta oscillation phasedependent modulation of TMS-induced motor responses in the motor cortex ${ }^{51}$. (3) Temporal biasing of spikes. Strong rhythmic applied fields may reliably affect the membrane potential and, consequently, spike timing of subsets of neurons. This mechanism is related to stochastic resonance since the coincidence of intrinsic and extrinsic polarization forces work together but the same neurons are more reliably activated from trial to trial. Such phase-locked temporal biasing of neuronal spikes by the rhythmic

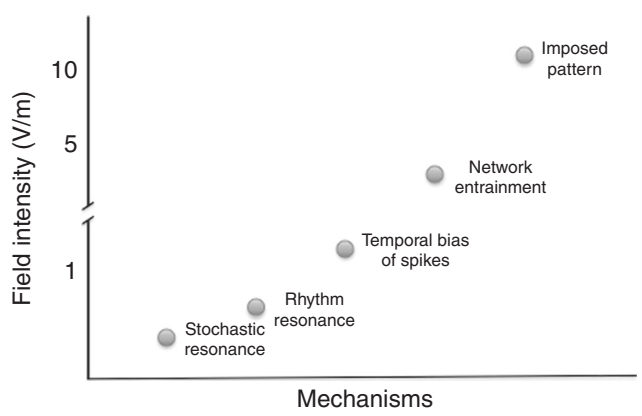

Fig. 2 Five postulated mechanisms to affect online spiking of neurons and networks patterns in response to different estimated magnitudes of TES. While the figure illustrates distinct effects, in reality the boundaries of mechanisms are blurred under most experimental conditions. Several mechanisms can act simultaneously in different networks of the same brain. The numbers on the vertical axis are merely estimates based on current data and theoretical considerations 
forced field may occur without affecting the overall firing rates of neurons. (4) Network entrainment. To entrain network patterns of less regularity (e.g., slow oscillations of sleep) requires stronger currents since exogenous patterns compete with native brain rhythms ${ }^{19,40,45}$. (5) Imposed Pattern. Imposing an arbitrary pattern on a neuronal network (e.g., enforcing theta activity on a network with an endogenous beta rhythm) requires the strongest field to overcome the endogenous control of network neurons (Fig. 2).

Spike and local field potential (LFP) measurements are oftenused methods of measuring neural activity in vivo, each with advantages and disadvantages. Spike measures are the most direct read-out of TES effect on neural activity ${ }^{35}$, but are not always available in animals and are rarely possible in humans. LFP is the extracellular measure of transmembrane electric current generated synchronously by a nearby population of neurons, brought about primarily by postsynaptic potentials ${ }^{52,53}$ (see Box 1). Importantly, LFP measurements allow comparison between human and animal experiments. The acute effects of tACS could be measured by changes in the LFP amplitude, such as epileptiform spike-and-waves or the dominant alpha rhythm as a function of the tACS phase $\mathrm{e}^{35,54}$. A related method is to measure entrainment of slower rhythms by tACS (e.g., sleep slow oscillations, see Box 1), also measured by the amplitude of faster, crossfrequency coupled patterns (e.g., sleep spindles) ${ }^{17,25}$ Quantifying entrainment of rhythms at the same frequency of the applied TES is difficult with current methods due to the difficulty of separating the artifact from the entrained physiological pattern, as discussed in more detail below.

\section{Acute effects of electric fields in vitro}

In brain slices, the effects of induced fields can be studied with exquisite control, since all synaptic signaling can be blocked pharmacologically ${ }^{55}$. Most studies apply constant fields across parallel wires outside the slice. This spatially diffuse stimulation differentially affects the neuronal compartments (e.g., soma, apical dendrites), producing complex effects ${ }^{23}$. In vitro recordings of rat hippocampal and neocortical pyramidal cells show that DC electric fields of $1 \mathrm{~V} / \mathrm{m}$ can polarize the soma of pyramidal neurons by $\sim 0.2 \mathrm{mV}$.

Yet, extracellular electric fields abound in the brain ${ }^{22}$. Local electric fields $(>0.5 \mathrm{~V} / \mathrm{m})$ applied in vitro with a micropipette near layer 5 pyramidal cell dendrites and soma induce small intracellular voltage fluctuations (e.g., $0.2 \mathrm{mV}$ ) and may phaselock the spikes to the low-frequency $(1-30 \mathrm{~Hz})$ oscillating external field without affecting overall spiking rate ${ }^{56}$. Thus, under idealized conditions, very weak but localized extracellular fields can bias neuronal activity (Fig. 3). Spike timing is highly sensitive to small, persistent oscillations that act throughout a volume. This voltage fluctuation is comparable to intrinsic, non-synaptic, internally generated noise in layer 5 pyramidal neurons $(0.2-0.4$ $\mathrm{mV})^{57}$ and is much smaller than synaptic background activity in vivo ${ }^{33,58}$. For example, hippocampal theta oscillations, for instance, induce $\sim 4 \mathrm{~V} / \mathrm{m}$ electric fields across the CA1 pyramidal layer, while hippocampal sharp waves induce $5-15 \mathrm{~V} / \mathrm{m}^{39}$. Endogenous field strengths associated with slow oscillations in the neocortex are $1-2 \mathrm{~V} / \mathrm{m}^{45}$. Whether weak applied electric fields can overcome such strong endogenous fields remains an important experimental question.

AC fields of sufficient strength applied at low frequencies $(<10 \mathrm{~Hz})$ can modulate firing rate periodically by the same mechanisms as DC fields ${ }^{59}$. For higher AC stimulation frequencies, the induced effects are diminished; for example, somatic polarization is reduced by $\sim 70 \%$ when a $50 \mathrm{~Hz}$ AC stimulation induces a $1 \mathrm{~V} / \mathrm{m}$ field, because the membrane cannot follow the rapid fluctuations in the electric field ${ }^{60}$. The oscillatory fields may a

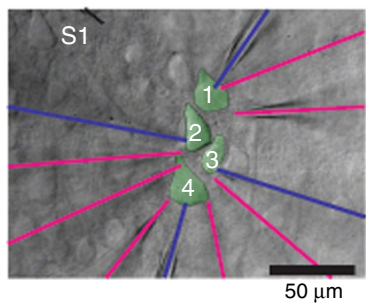

C

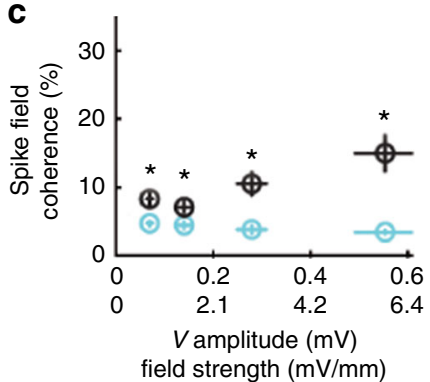

b

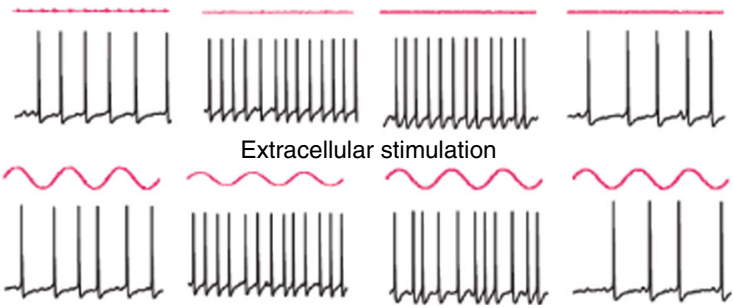

d

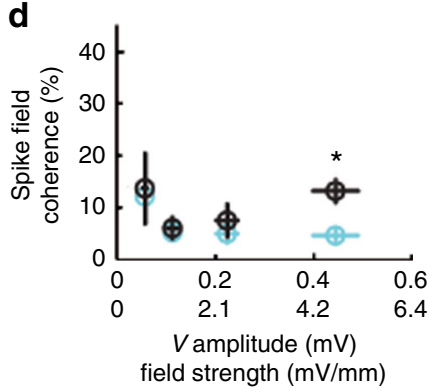

Fig. 3 Field-entrainment of spikes under idealized conditions in vitro. Synaptic transmission was blocked pharmacologically. a Four neurons with somata located within $100 \mu \mathrm{m}$ of tissue were patched with intracellular electrodes (blue). Seven extracellular electrodes monitored extracellular voltage (Ve) fluctuations (magenta). An extracellular stimulation electrode (S1) was placed 50-80 $\mu$ m from the recorded somata. b Each of the four intracellularly recorded neurons were depolarized to induced spiking. Spiking in the absence (top traces) and in the presence of extracellular stimulation (magenta; $100 \mathrm{nA}$ at $1 \mathrm{~Hz}$; bottom traces) is shown. c Spike field coherence (circles, mean; error bars, s.e.m.) between spikes and extracellular Ve is shown during $1 \mathrm{~Hz}$ extracellular stimulation (black) and control condition (cyan; circles indicate mean Ve amplitude at the soma and error bars indicate s.e.m.) as a function of stimulation strength. Asterisks indicate statistical significance of the spike-field difference between control and extracellular stimulation. d Spike-spike coordination (spike-field coherence for two simultaneously occurring spikes; essentially spike synchrony among neurons) during 1 $\mathrm{Hz}$ extracellular stimulation (black) and control (cyan) condition. Note that stronger fields are needed for coherent entrainment of neuronal spikes across the four neurons (d) than for inducing spike-field coherence for each neuron separately (c). Figure reproduced with permission 56 
not affect the overall firing rate but can modulate the timing of ongoing spikes ${ }^{56,59}$ (Fig. 3). AC stimulation at a frequency matching the resonant neuronal properties can induce cumulative effects over multiple cycles ${ }^{43,45,59-62}$. Under such ideal conditions, AC fields as low as $0.2-0.5 \mathrm{~V} / \mathrm{m}$ can shift spike timing $23,43,59,60$. Higher stimulation frequencies require stronger currents to bias spike-LFP coupling $(>0.7 \mathrm{~V} / \mathrm{m}$ induced field at 1 $\mathrm{Hz}$ and $>5 \mathrm{~V} / \mathrm{m}$ at $30 \mathrm{~Hz}^{56,39}$, Fig. 3), due to the frequency filtering properties of the neuronal membrane ${ }^{63}$. One caveat of these in vitro studies is that pharmacologically or intracellularly induced oscillations are much more regular than in vivo oscillations ${ }^{19}$. Overall, in vitro studies have laid the biophysical foundation for TES, providing quantitative relationships between the effective magnitude of voltage gradients for both $\mathrm{V}_{\mathrm{m}}$ and spiking activity. These experiments have also clarified the geometric relationship between the vectorial field and neuronal morphology and soma-dendritic orientation. However, these studies do not reveal how applied current affects neurons when applied through the skin, subcutaneous tissue, skull, dura and cerebrospinal fluid in the living animal. For a detailed summary of in vitro experiments measuring acute physiological changes during TES, see Supplementary Table 1 .

\section{Acute effects of external electric fields in rodents}

In rodent experiments, electric fields are often applied with electrodes placed on the skull, the dura mater, or directly on the brain surface. Often, a saline-filled cup is applied at various epicranial locations with a return electrode on the thorax or the contralateral hemisphere. Several in vivo studies have found a dose-dependent relationship between the induced electric field and spiking rate $^{24,34,35,40}$, transmembrane potential and, at higher stimulation intensities, on the LFP ${ }^{25,35,39}$. Applied electric fields can alter neuronal excitability ${ }^{64}$ and evoked responses ${ }^{64,65}$ in a polarity-specific manner.

However, rodent studies typically use ten-fold stronger current intensities compared to human studies ${ }^{66}$. Across the 28 rodent experiments reviewed ${ }^{67}$ (Supplementary Table 2), the induced intracranial fields averaged $6.8 \pm 3.8 \mathrm{~V} / \mathrm{m}(n=11$, ten epicranial and 1 subdural studies), compared to $<1 \mathrm{~V} / \mathrm{m}$ measured in conventional human TES studies ${ }^{19,68,69}$. While stimulating transcranially in rats, the lowest electric field sufficient to affect the timing of spiking activity in widespread cortical and hippocampal areas was $\sim 1 \mathrm{~V} / \mathrm{m}$; higher intensities were required to reliably affect LFP and the membrane potential in intracellularly recorded neurons in vivo ${ }^{35,40}$. Higher field intensities were also required in urethaneanesthetized rats to affect LFP oscillations ${ }^{70,71}$. Similarly, applied currents have terminated thalamocortical spike and wave patterns in rodents $(1.5-2 \mathrm{~mA} \text {, applied to skull })^{54,72}$ or triggered paw movement $(0.25 \mathrm{~mA} \text { and } 0.9 \mathrm{~mA})^{34}$ and modulated endogenous slow wave initiation and propagation patterns $\left(\sim 3 \mathrm{~mA} / \mathrm{mm}^{2}\right)^{73}$. In summary, rodent stimulation studies demonstrate physiological effects on spike timing, LFP oscillations, and terminating seizure patterns, but applied currents were several-fold stronger than the currents expected to penetrate the human brain using TES. For a detailed summary of in vivo experiments measuring acute physiological changes during TES, see Supplementary Table 2.

\section{Immediate electrophysiological effects of TES in primates}

Non-human primates permit TES investigations using a relatively large head with a gyrated cortical surface. The variable anatomy, including thick skull and large musculature, can result in a wide range of field strengths across studies and even across individuals. Opitz et al. ${ }^{69}$ measured field strengths in two anesthetized cebus monkeys with small round scalp electrodes $\left(3.14 \mathrm{~cm}^{2}\right)$ over the left occipital and frontal cortices stimulated with 1-2 mA. The median induced electric fields were $0.21 \mathrm{~V} / \mathrm{m}$ (strongest $0.36 \mathrm{~V} /$ $\mathrm{m}$ ) in a male monkey $(4.1 \mathrm{~kg}$ ) and $0.39 \mathrm{~V} / \mathrm{m}$ (strongest $1.16 \mathrm{~V} / \mathrm{m}$ ) in a female monkey $(2.9 \mathrm{~kg})$. The weaker effects in the male likely reflected the larger head musculature that shunted much of the applied current.

Krause et al. ${ }^{74}$ studied the effects of tDCS with intracranial electrodes in right prefrontal and left inferotemporal cortices in macaque monkeys performing an oculomotor foraging task. Based on a finite-element model ${ }^{75}, 2 \mathrm{~mA}$ anodal stimulation applied to the right prefrontal region induced field strengths between $0.4-0.7 \mathrm{~V} / \mathrm{m}$. Although $\mathrm{tDCS}$ did not affect the spontaneous or task-related firing rates of isolated single units or multi-unit clusters, LFP power in the $1-15 \mathrm{~Hz}$ band increased ten-fold during DC stimulation, and coherence within the prefrontal array increased at all frequencies from $2-100 \mathrm{~Hz}$. However, the changes in spike frequency or spikefield coherence in areas with stronger fields (up to $1 \mathrm{~V} / \mathrm{m}$ ) but outside the recording area, could not be ruled out.

Kar et al. ${ }^{76}$ delivered tACS of $1 \mathrm{~mA}$ peak amplitude at $10 \mathrm{~Hz}$ frequency through scalp electrodes placed anterior to the vertex and lateral to the middle temporal area (MT). In one monkey, the induced field was $0.12 \mathrm{~V} / \mathrm{m}$ at closely spaced electrodes $(0.5 \mathrm{~mm})$ in the recorded MT area. Spiking activity during the stimulation was not reported, but a reduction in spike adaptation to the visual input after tACS offset was observed.

In a dose-ranging $\mathrm{tDCS}$ experiment on motor control, Lee et a ${ }^{77}$. applied anodal stimulation over primary motor cortex (with cathode at the vertex) to determine the threshold to elicit a muscle twitch in sedated macaques. Between 50-120 mA ( $\sim 35-55 \mathrm{~V} / \mathrm{m}$ electric fields in the motor cortex) were needed to trigger a visible twitch.

In summary, primate models offer the advantage of testing TES in a larger head model with a gyrated cortical surface. The few primate studies which have been performed to date employ different montages and measurement techniques. Compared to the rodent models, primate experiments are limited by weaker induced fields and proportionately less cortical recording coverage. Primates with large heads, thick skulls, and greater musculature have greater shunting, or current loss away from the brain, and require larger current intensities to achieve comparable electric field strengths to those in rodent brains. These differences highlight the critical need to match intracerebral fields rather than stimulation parameters across species and experiments.

\section{Ex vivo human studies}

Translating the results of in vitro and in vivo animal experiments to humans is confounded by complexities of brain and head anatomy. Neurons in highly gyrated cortices of higher primates have variable alignments even on the convexity 29,78 , in contrast to those of lissencephalic rodents which are mostly perpendicular to the skull surface. Since entrainment efficacy of neurons depends on how polarized neurons align with the direction of the electrical field, TES of human cortical gyri may lead to unpredictable effects. Further, safety considerations require TES application through the hair, skin, and skull. In contrast, few animal experiments applied TES through the scalp ${ }^{35,69,74,76}$. Using transcranially applied stimulation, only a small fraction of the applied current enters the brain: most is lost through the skin, subcutaneous soft tissue, and the skull ${ }^{35}$ (Fig. 4a). One study conducted in rodents and human cadavers demonstrated that $>$ $75 \%$ of applied TES current is lost through shunting ${ }^{35}$, although the shunting effects differ between humans, nonhuman primates and rodents in unknown ways.

Human cadavers yield insights regarding conductivity and anisotropy. Multiple intraparenchymal, three-dimensional measurements of the electric field distribution can be obtained and multiple stimulation parameters can be tested in the same cadaver 
a
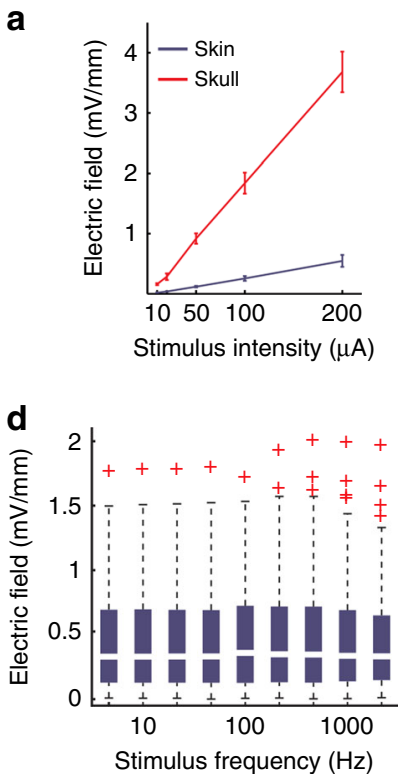

b

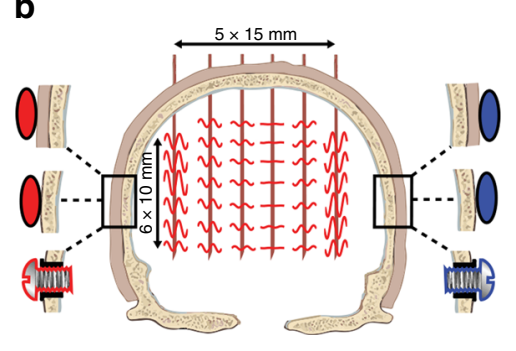

e

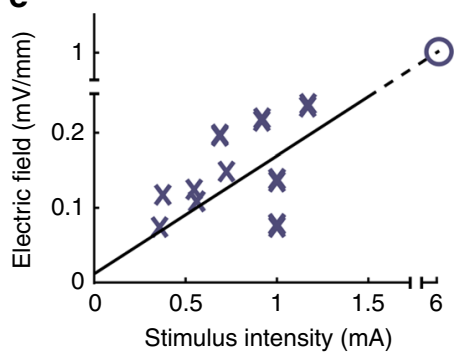

C

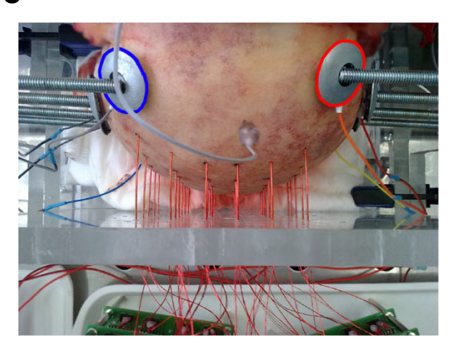

f

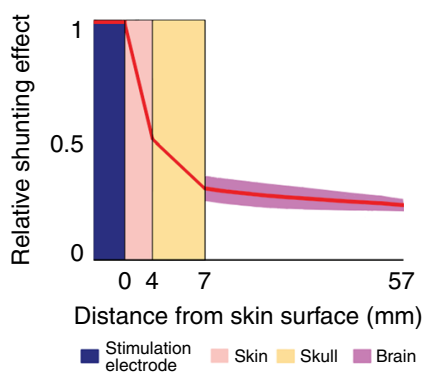

Fig. 4 Current loss of TES in rodents and in human cadaver brains. a Compared to subcutaneous stimulation (red), transcutaneous stimulation (blue) generated several-fold weaker electric fields in a rodent model. b Schematic of the experimental arrangement for transcutaneous, subcutaneous, and epidural stimulation in cadavers, in a coronal plane. c Photograph of the cadaver skull with drilled holes and inserted matrix of recording electrodes. Stimulation electrodes, marked by blue and red circles for negative and positive polarity, respectively, were fixed to the skull. d Effect of stimulus frequency on intracerebral voltage gradients. Stimulus frequency between 5 and $1000 \mathrm{~Hz}$ has a minor effect on intracerebral gradients. e Extrapolation from measurements in human cadavers (blue crosses) suggests that approximately $6 \mathrm{~mA}$ current applied across the skin would induce $1 \mathrm{~V} / \mathrm{m}$ intracerebral electric field (blue open circle). f Attenuation of charge flow (red line) through scalp (pink), skull (yellow), and brain (dark pink), as measured in human cadaver heads. Figure adapted from Figs. 1, 4, 5 in Voroslakos et al., (2018) ${ }^{35}$, under the Creative Commons License

(Fig. 4b, c). Such empirical measurements help determine the basic biophysical tissue properties and help model effects of TES into the brain parenchyma ${ }^{79-81}$ (Fig. $4 \mathrm{~d}-\mathrm{f}$ ). Intracerebral electric fields were measured in intact cadaver brains at 198 sites in a three-dimensional grid, while applying stimuli that varied in frequency, intensity, phase relationship, electrode location, and $\operatorname{size}^{35}$. The authors concluded that scalp, skull, brain, and cerebrospinal fluid behave as ohmic conductors. In other words, induced fields increased linearly with stimulation intensity, but were independent of stimulation frequency ${ }^{82}$. The comparison of transcutaneous, subcutaneous and epidural stimulations of the cadaver brain led to the conclusion that the bilateral transcutaneous stimulation of the cadaver heads with traditional montages and intensity (peak $1 \mathrm{~mA}$ ) induced only $\sim 0.2 \mathrm{~V} / \mathrm{m}$ field in the brain $^{35}$. Of note, death imparts profound changes in the biophysical properties of brain tissue, limiting a direct ex vivo to in vivo comparison ${ }^{83}$.

\section{In vivo human studies}

A critical question is the magnitude of the electric field reaching the brain when using conventional TES, applied transcutaneously at peak intensity currents of $1-2 \mathrm{~mA}$, because field strength constrains the expected nature of the physiological changes (Fig. 2). The main barrier for TES to reach the brain is the skull's high resistance $(\sim 160 \Omega \mathrm{m})$ combined with the low resistance of the scalp $(\sim 2 \Omega \mathrm{m})^{80}$. A large fraction (up to $75 \%$ ) of the current seeks the path of least resistance and shunts across the scalp ${ }^{35}$. Three independent studies using intracranial electrodes in epilepsy patients undergoing surgical evaluation demonstrated that maximal electric fields at the cortical surface under the electrodes were $<0.5 \mathrm{~V} / \mathrm{m}$ for $1 \mathrm{~mA}$ peak intensity currents ${ }^{68,69,84}$.
Can such weak electric fields reaching the human brain induce changes in neuronal networks, as measured by changes of the LFP? Oscillations are ubiquitous in the human brain, ranging from ultraslow $(0.05 \mathrm{~Hz})$ to ultrafast $(500 \mathrm{~Hz})$, with specific oscillation frequencies characterizing different arousal states or cognitive processes and coordinating activity between local and distant brain regions ${ }^{85}$. A leading hypothesis about the behavioral effect of tACS is that the applied stimulation frequency matching the dominant network oscillation entrains and augments endogenous rhythms ${ }^{37,38,62,86-89}$, such as gamma $60,89,90$, beta ${ }^{91}$, alpha $^{46}$, theta ${ }^{92}$, and slow frequency oscillations ${ }^{40,45,93}$.

Whether the weak electric fields resulting from TES at conventional intensities (1-2 mA peak) can entrain neuronal oscillations is technically difficult to assess. One technical challenge is that the magnitude of the stimulation artifact is dramatically larger than the magnitude of the endogenous oscillation, making artifact rejection difficult. To illustrate, supposing the use of a $10 \mathrm{~Hz}$ tACS waveform to entrain endogenous alpha oscillations, the amplitude of alpha waves is $\sim 100 \mu \mathrm{V}$ over the parietaloccipital lobes, whereas the artifact induced by a $2 \mathrm{~mA}$ tACS stimulus can exceed $1 \mathrm{~V}(\sim 1000 \mathrm{x}$ greater $)$. Therefore, even if $99.9 \%$ of the artifact is removed, the remaining $0.1 \%$ of the artifact would be as large as the endogenous alpha rhythm. Further, the stimulus waveform can be distorted, resulting in multiple harmonics of the applied signal. Due to non-linearities and instability of the stimulation waveform during the experiment, the waveform of the stimulation artifact and its harmonics across trials cannot simply be subtracted from the resulting EEG recordings. While artifact rejection algorithms can clean the recorded signals, artifact-free brain activity is unlikely to be recovered when the stimulation frequency matches the endogenous oscillation frequency ${ }^{94}$. Another challenge is the 
saturation of recording amplifiers, which precludes analysis of the endogenous LFP during stimulation.

Besides neurophysiology, other experimental measures of TES immediate effects include blinded subject reporting, TMS-evoked motor evoked potential (MEP), and fMRI. Feurra et al ${ }^{49}$ applied at $1.5 \mathrm{~mA}$ anodal current at varying frequencies of $(2-70 \mathrm{~Hz})$ over somatosensory cortex and asked subjects to report tactile sensation in the contralateral hand. While no sham or control stimulation site was included, during one of the two replications, subjects reliably reported sensation during alpha $(10-14 \mathrm{~Hz})$ and high gamma $(52-70 \mathrm{~Hz})$ frequency stimulation. These occasional behavioral effects of TES may reflect stochastic resonance (Fig. 3).

A study of TMS-evoked MEPs during tACS (1 mA peak-topeak) at different frequencies (theta, alpha, beta, and gamma) over the primary motor cortex during rest and during a motor imagery task found MEPs were greatest during beta frequency tACS at rest, but during theta-tACS during motor imagery ${ }^{38}$. Guerra et al. ${ }^{51}$, found beta frequency $(1 \mathrm{~mA}$ peak-to-peak, $20 \mathrm{~Hz})$ tACS stimulation acutely modulated the MEP amplitude in a phase-dependent manner; however another experiment ${ }^{37}$ (1.5 mA peak-to-peak) did not find phase-dependent modulation of corticospinal responses using low-frequency tACS $(0.8 \mathrm{~Hz})$ or tDCS superimposed with $0.8 \mathrm{~Hz}$. An fMRI study applied $20 \mathrm{~Hz}$ tACS stimulation ( $1 \mathrm{~mA}$ peak-to-peak) over the primary motor cortex (M1) found enhanced local connectivity within M1, without changing the overall local activity or long-range connectivity within the default mode network ${ }^{95}$. While these TMS and fMRI findings are consistent with a rhythm-resonance mechanism (i.e., $20 \mathrm{~Hz}$ modulation of resting beta frequency in motor cortex; Fig. 2), a peripheral contribution remains possible. Both consciously detected and subthreshold (unconscious) sensory stimuli can influence neocortical activity ${ }^{96,97}$.

Epilepsy patients undergoing invasive monitoring offer the opportunity to measure and analyze endogenous oscillations during TES and thus directly monitor brain responses, since intracranial signals are an order of magnitude larger than those recorded from the scalp. The effects of tACS $(0.75 \mathrm{~Hz}$ and $1 \mathrm{~Hz})$ during NREM sleep and waking rest were assessed in epilepsy surgery patients at standard stimulation intensities (up to $2.5 \mathrm{~mA}$ peak to peak; maximum induced field: $0.43 \mathrm{~V} / \mathrm{m}$ ). During NREM sleep, slow oscillations $(\sim 1 \mathrm{~Hz})$ strongly entrain spindle activity in the $10-15 \mathrm{~Hz}$ range. This provides the opportunity to test whether endogenous slow-wave rhythms can be entrained with low frequency $(0.75 \mathrm{~Hz}$ or $1 \mathrm{~Hz}) \mathrm{tACS}$, while measuring entrainment in a different frequency band that is largely uncontaminated by stimulation artifacts. While spindle and gamma activity robustly entrained to the phase of the endogenous slow oscillation, lowfrequency tACS failed to entrain spindle or gamma activity at over 1000 electrode sites measured in patients during NREM sleep. Likewise, no entrainment of gamma or theta activity was observed during waking rest ${ }^{19,98}$.

Instead of entrainment, TES-phase/EEG-amplitude coupling can also be examined with scalp recordings. This approach was validated by concurrent unit recordings in rat experiments ${ }^{54}$. In agreement with the intracerebral recordings ${ }^{19}$, no detectable effect was obtained on the scalp EEG when TES was applied at $<$ $2.5 \mathrm{~mA}$ in healthy subjects ${ }^{35}$. However, reliable tACS phase modulation of the amplitude of alpha rhythms was present when intensities exceeded 4.5-6 mA. The effect was specific for neuronal stimulation of cortical neurons because alpha amplitude enhancement over the left and right occipital cortex occurred in phase with alternating the anodal stimulation. Furthermore, to rule out peripheral contributions, control experiments applying stimulation to the abdomen did not produce an effect on alpha activity.

\section{Novel TES methods}

Current applied through the scalp stimulates electrically sensitive elements from the scalp surface to the brain. These peripheral side effects, including stimulation of cranial nerves, retina, and vestibular system, limit the maximum tolerable dosing in humans using traditional TES approaches. Currents exceeding 1-2 mA (depending on electrode type) can cause itching, burning sensation, pain at the skin under and around the electrodes ${ }^{99}$.

The next generation of TES technologies should make three advancements: (1) delivery of stronger currents to the brain while minimizing peripheral and indirect effects; (2) simultaneous stimulation and recording of brain activity for quantitative measurements of TES-induced effects; and (3) targeting of specific rhythms through closed-loop stimulation of brain areas, including deep brain structures.

Multi-electrode stimulation is one potential method to increase current density and focality of stimulation delivered to the brain surface. The strongest transmembrane polarization is expected to build up where electric fields overlap inside the head, whereas the intensity of local scalp stimulation is divided proportionally by the number of stimulation electrode pairs. The approach is similar to previous efforts which used multiple small electrodes to optimally target specific brain areas ${ }^{100}$

Two other approaches attempt to increase the brain to scalp stimulation ratio. The first is temporal interference stimulation. First introduced for stimulating peripheral nerves and brain ${ }^{101}$, temporal interference is now routinely used in physical therapy 102 . When applied to the mouse brain, intersecting $2 \mathrm{kHz}$ and $2.01 \mathrm{kHz}$ sinusoidal waveforms yielded a $10 \mathrm{~Hz}$ amplitude modulation of the LFP ${ }^{34}$. Because the high-frequency stimulus exceeds the time constant of the peripheral nerve membrane, the fast alternating current may reduce skin sensations and avoid retinal responses, while the interfering signals in the brain are expected to be strong enough to modulate neuronal firing. The second approach, 'intersectional short pulse' (or ISP) stimulation, uses multiple electrode pairs ${ }^{35}$. This time-multiplexing method exploits the time integrating property of the neuronal membrane (i.e., the membrane time constant of cortical neurons is $\sim 30 \mathrm{~ms}$ ), by applying currents that switch every $60 \mu$ s between the electrode pairs. The strongest transmembrane charge will build up where successively induced electric fields overlap inside the head, whereas the intensity of local scalp stimulation is divided proportionally by the number of stimulation electrode pairs. Again, the more electrode pairs are used, the greater the potential to generate brain focal stimulation while minimizing stimulation of the scalp to reduce skin sensations ${ }^{78,100}$. Initial studies using temporal interference and ISP show increased spatial targeting in animal models, but further analysis and refinements of these techniques are necessary.

The application of high-frequency pulses avoids the saturation of recording amplifiers and allows for the simultaneous measurement of the direct physiological effects of tES in humans ${ }^{35}$. Amplifier modifications, such as front-end subtraction of the applied tES waveform can further reduce induced artifacts. Such innovations are essential to directly quantify the physiological responses of neuronal circuits.

\section{Recommendations for future experiments}

Although the lasting effects of stimulation are the main purpose of most clinical trials ${ }^{103,104}$, our review focused on the immediate physiological effects of TES on spikes and network dynamics. A more accurate and sophisticated understanding of physiology, grounded in empirical measurements, will improve design and execution of future TES experiments, with more potent and 
consistent clinical benefits. Below, we offer a list of tasks for future TES research.

Exploration of peripheral and non-neuronal mechanisms of TES. It is tacitly or explicitly assumed that behavioral and clinical effects of TES are mediated by directly affecting neuronal activity in the brain. However, behavioral and cognitive changes in response to scalp electric stimulation may be mediated by other effects as well. Electric fields affect the excitability of subcutaneous nerves which signal to the brain. Even when the subject does not report a conscious experience of TES, sensory stimulation can still indirectly affect brain circuits ${ }^{97,105}$. Therefore, control experiments that measure the conscious and subconscious effects of sensory stimulation should be included in future studies. This can be achieved by including an "active control," such as stimulation of the neck or abdominal areas $^{35}$ or application of TES to another region of the brain ${ }^{106}$. In addition, applying topical anesthesia to the scalp under the electrodes and testing varying intensities of skin stimulation could exclude or characterize sensory contributions.

Long-lasting TES effects can be indirectly mediated by nonneuronal mechanisms as well, including trophic factors ${ }^{107}$, neurotransmitter metabolism ${ }^{51,108}$ glia $^{109,110}$, neuroendocrine system $^{111}$, fibroblasts ${ }^{112}$ lymphocytes, and other electrically charged immune system components ${ }^{113}$. These potential nonneuronal effects should be considered with low-intensity scalp stimulation, which may be too weak to induce the necessary electric field strengths to instantaneously affect neuronal activity. These indirect effects may play a role even when neuronal activity is directly affected by stronger TES.

Targeting neural circuits. Growing evidence, summarized above, suggests that TES applied at $\pm 1 \mathrm{~mA}$ peak intensity ( $2 \mathrm{~mA}$ peak to peak) generates $<0.5 \mathrm{~V} / \mathrm{m}$ electric fields in the human brain. This is sufficient to generate $0.1-0.2 \mathrm{mV}$ changes in the membrane potential of cells within the stimulated area. As these changes are significantly lower than the $20 \mathrm{mV}$ depolarization required to bring a neuron from its resting potential to spike threshold in vitro, TES is unlikely to directly elicit changes in spiking activity. Instead, weak induced fields may be more effective when used to bias or augment ongoing rhythms instead of introducing new activity patterns. Applying current at the optimal phase of endogenous rhythms in a closed-loop system may be most effective $^{54}$. However, such responsive methods require characterization of the affected circuitry and continuous monitoring and adjustment of the relevant rhythms ${ }^{114}$. This may be achieved with weak electric fields but other applications may require higher field strengths. For example, prompt control of spiking activity (e.g., to terminate a seizure) may require field strengths > $5 \mathrm{~V} / \mathrm{m}^{72}$.

Modeling for spatial targeting and to increase replication across subjects and studies. Individual variations in neuroanatomy (scalp, muscle, and skull thickness) can significantly alter the strength and distribution of the induced electrical field in humans ${ }^{68,98,115-117}$ and experimental animals ${ }^{75,77}$, and thus may account for inconsistent behavioral results in human TES experiments. This is exemplified by Fig. 5, which compares two individual models, with the same surface electrode montage but different head anatomy (Fig. 5a, b, created using previously published data and methods $\left.{ }^{18,119}\right)$. Differences in skull thickness and cerebrospinal fluid volume in posterior regions lead to different field distributions (Fig. 5a, b).

Computational models of current flow have been continuously refined over the last decade $78,100,116,117,120-122$. Recent head models can improve spatial targeting by guiding stimulation electrode placement (Fig. 5c). These models are based on segmentation of tissue (at $1 \mathrm{~mm}^{3}$ resolution) into compartments of differing electrical conductivities, including skin and other soft tissues, skull, gray matter, white matter, ventricles, and cerebrospinal fluid. Models indicate that focal stimulation of a few centimeters may be achieved at the cortical surface ${ }^{100}$, generally with a trade-off between focality and intensity of stimulation (Fig. 5). Deep targets may be reached at relatively high field intensities when adjacent to CSF-filled ventricles, which can guide currents deep into the brain ${ }^{68}$.

Computational models can help optimize electrode locations for maximal stimulation of a desired brain target ${ }^{100}$. Individual MRI head anatomy combined with toolboxes such as ROAST, and SimNIBS, and SCIrun can define field distribution for this purpose. When an individual's head MRI is not available, validated and calibrated universal head models can help reproduce in vivo intracranial measurements ${ }^{118,119,123}$. Furthermore, multielectrode montages can be optimized to engage local and network level targets, as demonstrated by a technique combining electric field modeling and cortical networks derived from PET or resting state functional connectivity MRI ${ }^{124}$.

Human TES experiments typically report the stimulation protocols by reporting the stimulating electrode positions (e.g., "anode at F8" or "over left dorsolateral PFC") and current intensities (e.g., " $2 \mathrm{~mA}^{\text {") }}$. These specifications describe how the experiment was designed and performed, but they only indirectly define the induced electrical field strength and distribution. Reporting induced field estimates from head models would help relate behavioral and clinical reports to underlying neural mechanisms. When the individual MRI head is available, subject-specific estimates of the induced field strength should be included as a covariate in analyses of its behavioral or neural effects. Intensity-response curves, inverted polarity stimulations and skin stimulations far from the desired target location may distinguish between direct brain-stimulation and peripheral effects. Measurements or estimates of the electric field strength are included in some recent human and large animal studies but are missing from most rodent studies. Reporting field magnitudes is important for animal studies where variable head size and anatomy can strongly affect field magnitudes. These values would provide a critical translational scaling metric to facilitate comparisons with human and animal studies and should be included in future publications.

Hardware and signal processing issues. The simultaneous measurement of physiological activity (EEG, ECoG) during tACS is complicated by stimulation artifacts several orders of magnitude larger than intrinsic brain activity $(1-100 \mu \mathrm{V})$. When brain activity and stimulation are recorded simultaneously, special care should be taken to (1) avoid saturation of the recording amplifier during experiments, and (2) subtract stimulation artifacts from the measured signal. Saturation can be avoided by using highquality amplifiers with sufficient input dynamic range (e.g. an amplifier with dynamic range of $\pm 100 \mathrm{mV}$ can capture the full stimulation artifact).

Artifact rejection methods initially suggested for scalp $\mathrm{EEG}^{87}$ are highly unlikely to fully recover artifact-free brain activity (see above $)^{94,125}$. Therefore, when using tACS stimulation, it is difficult to analyze online neural responses at the same frequency in which stimulation is applied (e.g., increases in alpha oscillations during $10 \mathrm{~Hz}$ tACS stimulation). Analysis should target frequencies well away from the stimulation frequency and its harmonics. Measurements using phase-amplitude coupling or single-unit recordings may also be used when available. 
a
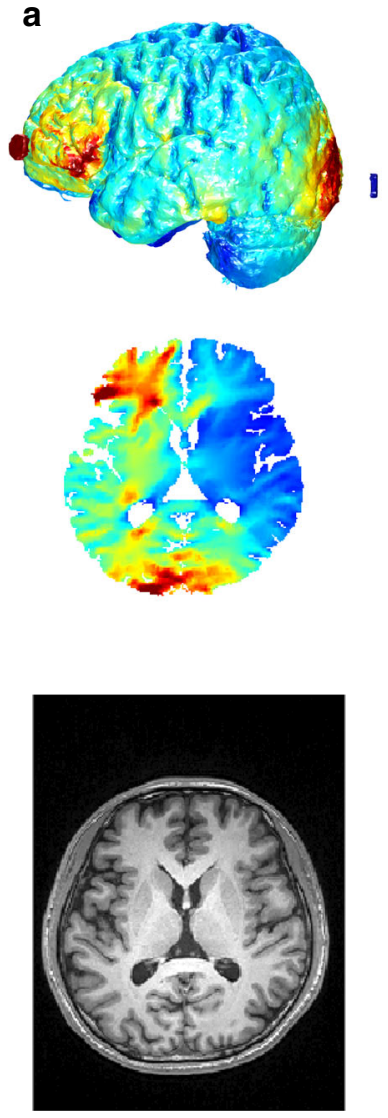

b
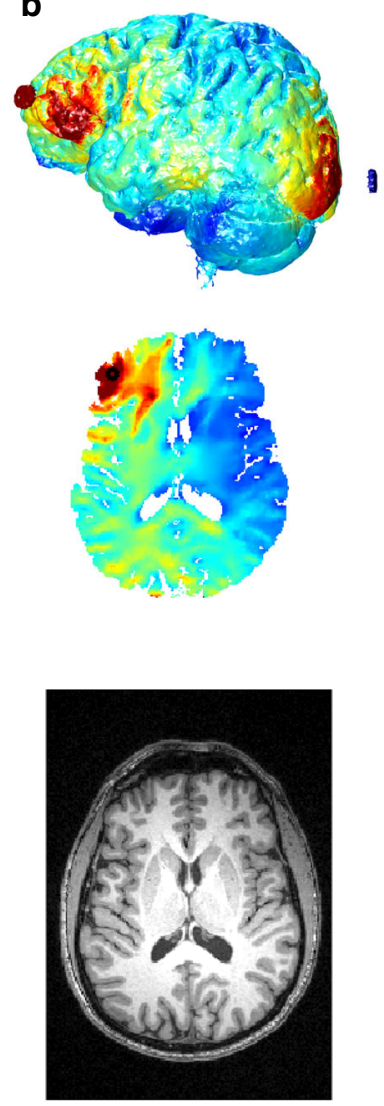

C
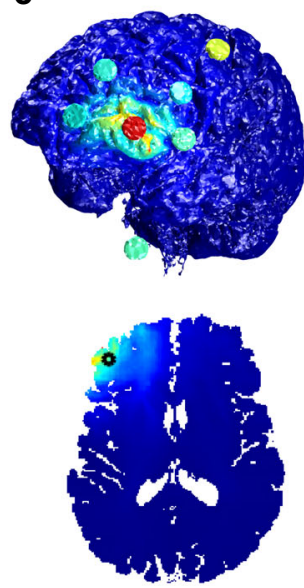

Injected current

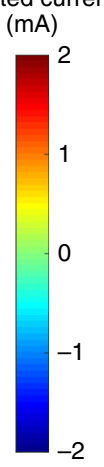

Electric field $(\mathrm{V} / \mathrm{m})$

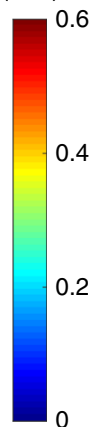

Fig. 5 Individualized models of transcranial electrical stimulation for two subjects with variable electrode placements. a Model of Subject 1 , with $2 \mathrm{~mA}$ current injected at electrode Fp1 and return from electrode P3. b Model of Subject 2 with the same electrode configuration. c Model of Subject 2 with another electrode configuration attempting to achieve more focal stimulation. Note that stimulation is more focal but achieves weaker fields compared to $\mathbf{b}$, due to increased current shunting through the skin between near-by electrodes. Electrode montages in $\mathbf{b}$ and $\mathbf{c}$ are obtained by a numerical optimization algorithm that attempts to achieve maximal intensity (b) or maximal focality (c) for the location indicated by a black circle. The current-flow models were generated from previously published data ${ }^{118}$ and methods ${ }^{119}$. We used ROAST, a toolbox for realistic current-flow models of the human ${ }^{119}$

DC stimulation artifacts pose less of an issue during signal processing as hardware filters will typically remove any DC offset (avoiding amplifier saturation). However, EEG recordings in humans are affected by artifacts in the $1-15 \mathrm{~Hz}$ frequency band due to heartbeats and eye-blink ${ }^{125,126}$. The effect is most prominent in frontal locations. In addition, care should be taken to avoid motion of the subject and stimulation electrodes, electrical noise injected into the system is reduced by using highquality (i.e., narrow band) stimulation devices, and environmental noise (ensure adequate electrical isolation ${ }^{19,68}$. Control experiments, e.g., using a phantom head model, are needed to separate TES-related artifacts from the induced neural activity.

Data sharing standards. Because different devices use different conventions, it is critical to specify whether tACS amplitudes describing the absolute magnitude (i.e., the amplitude of one phase), the peak-to-peak amplitude, or another form of parameterization. Shared data and analysis methods can facilitate coordinated community efforts and metanalysis. Combining many data sets may offer novel insights and interpretations (e.g., https://www.nwb.org/). Further, given the artifact rejection methods described above, sharing raw EEG data sets would enhance replication of the signal processing pipeline. Sharing analysis software packages will facilitate cross-validation of results across studies.

\section{Conclusions}

A more accurate and sophisticated understanding of TES physiology, grounded in empirical measurements, will improve the design and execution of neurostimulation experiments, yielding more robust and consistent clinical benefits. While the authors of this review share a consensus on experimental recommendations, we acknowledge a controversy within the TES field and amongst ourselves as to whether the attenuated electrical fields that reach the human brain with conventional protocols can directly affect neurons and neuronal networks. While direct neural mechanisms have been measured with exquisite experimental control in vitro and in vivo models, whether these insights translate to humans is uncertain. We have highlighted challenges in translating mechanistic insights from experimental models to humans, including (1) the interaction of the applied external field with competing or cooperating network oscillations when comparing in vitro to in vivo studies; (2) external fields applied to the folded cortical surface produce more variable and unpredictable effects in larger animals (including humans) compared to rodent and in vitro models; (3) the large human head and the low resistance scalp shunt most applied current, with much smaller fraction of the applied current reaching the cortical surface.

The weak induced electric fields reaching the human brain contrast with the numerous behavioral and clinical effects reported. We should also consider how TES can affect brain activity indirectly, including activation of afferent nerves ${ }^{127}$, 
retina and the vestibular apparatus ${ }^{42}$, astrocytes, perivascular elements, and glial activation 109,110 , as well as through placebo effects $^{128}$. Both tACS and tDCS may also induce cumulative or longer-term effects via unknown mechanisms that merit further exploration. Future human behavioral trials should control for sensory contributions with approaches such as topical anesthesia, or by using sensory controls such as stimulation away from the desired targets.

Novel stimulation methods that safely induce higher brain to scalp electric field ratios are needed. Existing experimental techniques, such as temporal interference stimulation and intersectional short pulse stimulation delivering stimulation at high frequencies $(>1 \mathrm{kHz})$, require further testing in both experimental animals and humans. These and related methods, such as multielectrode $\mathrm{TES}^{78,100}$, may better target brain structures compared to conventional electrode montages. Closed-loop techniques may enhance the efficacy of TES via resonant modulation of native brain rhythms. In selected cases, supported by experimental findings and human head-based modeling, subcutaneous arrays or electrode plates inserted below the skin may provide superior efficacy, similar to techniques to treat headache $^{129}$ and facial pain ${ }^{129,130}$ by stimulating peripheral nerves. With further development, TES could become a leading tool for chronic, on-demand, at-home treatment of many neuropsychiatric and neurological diseases.

Received: 26 April 2018 Accepted: 18 October 2018

Published online: 30 November 2018

\section{References}

1. von Helmholtz, H. Treatise on Physiological Optics. Vol. III, (Dover Publications, New York, 1867).

2. Rohracher, H. Ueber subjektive Lichterscheinungen bei Reizung mit Wechselströmen. Zeitschrift für Sinnesphysiologie 66, 164-181 (1935).

3. Barlow, H. B., Kohn, H. I. \& Walsh, E. G. Visual sensations aroused by magnetic fields. Am. J. Physiol. 148, 372-375 (1947).

4. Kar, K. \& Krekelberg, B. Transcranial electrical stimulation over visual cortex evokes phosphenes with a retinal origin. J. Neurophysiol. 108, 2173-2178 (2012).

5. Rudorfer, M., Henry, M. \& Sackeim, H. in Psychiatry (eds. Tasman, A., Kay, J. \& Lieberman, J.) 1539 (WB Saunders, Philadelphia, 1997).

6. Brown, C. C. Electroanesthesia and electrosleep. Am. Psychol. 30, 402-410 (1975).

7. Kuzin, M. I., Sachkov, V. I. \& Zhukovskiy, V. D. Electronarcosis produced by interference currents in clinical practice. Present-Day Technique in Surgery: Data of the Sixth Scientific Session of the Scientific Research Institute of Experimental Surgical Apparatus and Instruments. JPRS 31, 4,5 (1965).

8. Calancie, B. intraoperative neuromonitoring and alarm criteria for judging MEP responses to transcranial electric stimulation: the threshold-level method. Clin. Neurophysiol. 34, 12-21 (2017).

9. Merton, P. A. \& Morton, H. B. Stimulation of the cerebral cortex in the intact human subject. Nature 285, 227 (1980).

10. Rothwell, J., Thompson, P., Day, B., Boyd, S. \& Marsden, C. Stimulation of the human motor cortex through the scalp. Exp. Physiol. 76, 159-200 (1991).

11. Lang, J., Sances, A. \& Larson, S. J. Determination of specific cerebral impedance and cerebral current density during the application of diffuse electrical currents. Med. Biol. Eng. 7, 517-526 (1969).

12. Toleikis, J. R., Sances, A. Jr. \& Larson, S. J. Effects of diffuse transcerebral electrical currents on cortical unit potential activity. Anesth. Analg. 53, 48-55 (1974).

13. Nitsche, M. A. \& Paulus, W. Excitability changes induced in the human motor cortex by weak transcranial direct current stimulation. J. Physiol. 527(Pt 3), 633-639 (2000).

14. Nitsche, M. A. \& Paulus, W. Sustained excitability elevations induced by transcranial DC motor cortex stimulation in humans. Neurology 57, 1899-1901 (2001).

15. Fry, F. J., Ades, H. W. \& Fry, W. J. Production of reversible changes in the central nervous system by ultrasound. Science 127, 83-84 (1958).

16. Kasschau, M. et al. Transcranial Direct Current Stimulation Is Feasible for Remotely Supervised Home Delivery in Multiple Sclerosis. Neuromodulation 19, 824-831 (2016).
17. Liu, A., Fregni, F., Hummel, F. \& Pascual-Leone, A. in Transcranial Brain Stimulation (eds. Miniussi, C., Paulus, W. \& Rossini, P. M.). 359-414 (CRC Press, Boca Raton, 2012).

18. Horvath, J. C. Are current blinding methods for transcranial direct current stimulation (tDCS) effective in healthy populations? Clin. Neurophysiol. 126, 2045-2046 (2015).

19. Lafon, B. et al. Low frequency transcranial electrical stimulation does not entrain sleep rhythms measured by human intracranial recordings. Nat. Commun. 8, 1199 (2017).

20. Polania, R., Nitsche, M. A. \& Ruff, C. C. Studying and modifying brain function with non-invasive brain stimulation. Nat. Neurosci. 21, 174-187 (2018).

21. Stagg, C. J., Antal, A. \& Nitsche, M. A. Physiology of transcranial direct current stimulation. J. Ect. 34, 144-152 (2018).

22. Buzsaki, G., Anastassiou, C. A. \& Koch, C. The origin of extracellular fields and currents-EEG, ECoG, LFP and spikes. Nat. Rev. Neurosci. 13, 407-420 (2012).

23. Terzuolo, C. A. \& Bullock, T. H. Measurement of Imposed Voltage Gradient Adequate to Modulate Neuronal Firing. Proc. Natl Acad. Sci. USA 42, 687-694 (1956).

24. Creutzfeldt, O. D., Fromm, G. H. \& Kapp, H. Influence of transcortical d-c currents on cortical neuronal activity. Exp. Neurol. 5, 436-452 (1962).

25. Purpura, D. P. \& McMurtry, J. G. Intracellular activities and evoked potential changes during polarization of motor cortex. J. Neurophysiol. 28, 166-185 (1965).

26. Ranck, J. B. Jr. Which elements are excited in electrical stimulation of mammalian central nervous system: a review. Brain Res. 98, 417-440 (1975).

27. Bikson, M., Radman, T. \& Datta, A. Rational modulation of neuronal processing with applied electric fields. Conf. Proc. Ieee. Eng. Med. Biol. Soc. 1, 1616-1619 (2006)

28. Radman, T., Ramos, R. L., Brumberg, J. C. \& Bikson, M. Role of cortical cell type and morphology in subthreshold and suprathreshold uniform electric field stimulation in vitro. Brain Stimul. 2, 215-228 (2009).

29. Rahman, A. et al. Cellular effects of acute direct current stimulation: somatic and synaptic terminal effects. J. Physiol. 591, 2563-2578 (2013).

30. Chan, C. Y. \& Nicholson, C. Modulation by applied electric fields of Purkinje and stellate cell activity in the isolated turtle cerebellum. J. Physiol. 371, 89-114 (1986).

31. Katz, B. \& Schmitt, O. H. Electric interaction between two adjacent nerve fibres. J. Physiol. 97, 471-488 (1940).

32. Stagg, C. J. et al. Polarity-sensitive modulation of cortical neurotransmitters by transcranial stimulation. J. Neurosci. 29, 5202-5206 (2009).

33. Gutnick, M. J. \& Prince, D. A. Proceedings: spontaneous antidromic spikes from axons in cortical penicillin foci. Epilepsia 13, 354-355 (1972).

34. Grossman, N. et al. Noninvasive deep brain stimulation via temporally interfering electric fields. Cell 169, 1029-1041 (2017).

35. Voroslakos, M. et al. Direct effects of transcranial electric stimulation on brain circuits in rats and humans. Nat. Commun. 9, 483 (2018).

36. Antal, A. et al. Comparatively weak after-effects of transcranial alternating current stimulation (tACS) on cortical excitability in humans. Brain Stimul. 1, 97-105 (2008).

37. Bergmann, T. O. et al. Acute changes in motor cortical excitability during slow oscillatory and constant anodal transcranial direct current stimulation. J. Neurophysiol. 102, 2303-2311 (2009).

38. Feurra, M. et al. State-dependent effects of transcranial oscillatory currents on the motor system: what you think matters. J. Neurosci. 33, 17483-17489 (2013).

39. Anastassiou, C. A., Montgomery, S. M., Barahona, M., Buzsaki, G. \& Koch, C. The effect of spatially inhomogeneous extracellular electric fields on neurons. J. Neurosci. 30, 1925-1936 (2010).

40. Ozen, S. et al. Transcranial electric stimulation entrains cortical neuronal populations in rats. J. Neurosci. 30, 11476-11485 (2010).

41. Geisler, C. D. \& Goldberg, J. M. A stochastic model of the repetitive activity of neurons. Biophys. J. 6, 53-69 (1966).

42. Schutter, D. J. \& Hortensius, R. Retinal origin of phosphenes to transcranial alternating current stimulation. Clin. Neurophysiol. 121, 1080-1084 (2010).

43. Francis, J. T., Gluckman, B. J. \& Schiff, S. J. Sensitivity of neurons to weak electric fields. J. Neurosci. 23, 7255-7261 (2003)

44. Rosanova, M. et al. Natural frequencies of human corticothalamic circuits. J. Neurosci. 29, 7679-7685 (2009).

45. Frohlich, F. \& McCormick, D. A. Endogenous electric fields may guide neocortical network activity. Neuron 67, 129-143 (2010).

46. Zaehle, T., Rach, S. \& Herrmann, C. S. Transcranial alternating current stimulation enhances individual alpha activity in human EEG. PLoS ONE 5, e13766 (2010) 
47. Romei, V. et al. Causal evidence that intrinsic beta-frequency is relevant for enhanced signal propagation in the motor system as shown through rhythmic TMS. Neuroimage 126, 120-130 (2016).

48. Kanai, R., Chaieb, L., Antal, A., Walsh, V. \& Paulus, W. Frequency-dependent electrical stimulation of the visual cortex. Curr. Biol. 18, 1839-1843 (2008).

49. Feurra, M. et al. Frequency-dependent tuning of the human motor system induced by transcranial oscillatory potentials. J. Neurosci. 31, 12165-12170 (2011).

50. Riecke, L., Formisano, E., Herrmann, C. S. \& Sack, A. T. 4-Hz Transcrania alternating current stimulation phase modulates hearing. Brain Stimul. 8, 777-783 (2015).

51. Guerra, A. et al. Phase dependency of the human primary motor cortex and cholinergic inhibition cancelation during beta tACS. Cereb. Cortex 26, 3977-3990 (2016).

52. Mitzdorf, U. Current source-density method and application in cat cerebral cortex: investigation of evoked potentials and EEG phenomena. Physiol. Rev. 65, 37-100 (1985).

53. Nicholson, C. Theoretical Analysis of Field Potentials in Anisotropic Ensembles of Neuronal Elements. IEEE Trans. Biomed. Eng. BME 20, 278-288 (1973).

54. Berenyi, A., Belluscio, M., Mao, D. \& Buzsaki, G. Closed-loop control of epilepsy by transcranial electrical stimulation. Science 337, 735-737 (2012).

55. Jefferys, J. G. Influence of electric fields on the excitability of granule cells in guinea-pig hippocampal slices. J. Physiol. 319, 143-152 (1981).

56. Anastassiou, C. A., Perin, R., Markram, H. \& Koch, C. Ephaptic coupling of cortical neurons. Nat. Neurosci. 14, 217-223 (2011).

57. Jacobson, G. A. et al. Subthreshold voltage noise of rat neocortical pyramidal neurones. J. Physiol. 564, 145-160 (2005).

58. Nunez, A., Amzica, F. \& Steriade, M. Electrophysiology of cat association cortical cells in vivo: intrinsic properties and synaptic responses. $J$. Neurophysiol. 70, 418-430 (1993).

59. Reato, D., Rahman, A., Bikson, M. \& Parra, L. C. Low-intensity electrical stimulation affects network dynamics by modulating population rate and spike timing. J. Neurosci. 30, 15067-15079 (2010).

60. Deans, J. K., Powell, A. D. \& Jefferys, J. G. Sensitivity of coherent oscillations in rat hippocampus to AC electric fields. J. Physiol. 583, 555-565 (2007).

61. Herrmann, C. S., Murray, M. M., Ionta, S., Hutt, A. \& Lefebvre, J. Shaping intrinsic neural oscillations with periodic stimulation. J. Neurosci. 36, 5328-5337 (2016).

62. Thut, G., Miniussi, C. \& Gross, J. The functional importance of rhythmic activity in the brain. Curr. Biol. 22, R658-R663 (2012).

63. Vaidya, S. P. \& Johnston, D. Temporal synchrony and gamma-to-theta power conversion in the dendrites of CA1 pyramidal neurons. Nat. Neurosci. 16, 1812-1820 (2013).

64. Bindman, L. J., Lippold, O. C. \& Redfearn, J. W. Long-lasting changes in the level of the electrical activity of the cerebral cortex produced bypolarizing currents. Nature 196, 584-585 (1962).

65. Reato, D., Rahman, A., Bikson, M. \& Parra, L. Effects of weak transcranial alternating current stimulation on brain activity-a review of known mechanisms from animal studies. Front. Hum. Neurosci. 7, 687 (2013).

66. Esmaeilpour, Z. et al. Notes on human trials of transcranial direct current stimulation between 1960 and 1998. Front. Hum. Neurosci. 11, 71 (2017).

67. Jackson, M. P. et al. Animal models of transcranial direct current stimulation: Methods and mechanisms. Clin. Neurophysiol. 127, 3425-3454 (2016).

68. Huang, Y. et al. Measurements and models of electric fields in the in vivo human brain during transcranial electric stimulation. eLife 6, e18834 (2017).

69. Opitz, A. et al. Spatiotemporal structure of intracranial electric fields induced by transcranial electric stimulation in humans and nonhuman primates. Sci. Rep. 6, 31236 (2016).

70. Greenberg, A. \& Dickson, C. T. Spontaneous and electrically modulated spatiotemporal dynamics of the neocortical slow oscillation and associated local fast activity. Neuroimage 83, 782-794 (2013).

71. Greenberg, A., Whitten, T. A. \& Dickson, C. T. Stimulating forebrain communications: slow sinusoidal electric fields over frontal cortices dynamically modulate hippocampal activity and cortico-hippocampal interplay during slow-wave states. Neuroimage 133, 189-206 (2016).

72. Kozak, G. \& Berenyi, A. Sustained efficacy of closed loop electrical stimulation for long-term treatment of absence epilepsy in rats. Sci. Rep. 7, 6300 (2017).

73. Greenberg, A., Abadchi, J. K., Dickson, C. T. \& Mohajerani, M. H. New waves: rhythmic electrical field stimulation systematically alters spontaneous slow dynamics across mouse neocortex. Neuroimage 174, 328-339 (2018).

74. Krause, M. R. et al. Transcranial direct current stimulation facilitates associative learning and alters functional connectivity in the primate brain. Curr. Biol. 27, 3086-3096 (2017).

75. Datta, A. et al. On comparing in vivo intracranial recordings in non-human primates to predictions of optimized transcranial electrical stimulation. Conf. Proc. Ieee. Eng. Med. Biol. Soc. 2016, 1774-1777 (2016).
76. Kar, K., Duijnhouwer, J. \& Krekelberg, B. Transcranial alternating current stimulation attenuates neuronal adaptation. J. Neurosci. 37, 2325-2335 (2017)

77. Lee, W. H., Lisanby, S. H., Laine, A. F. \& Peterchev, A. V. Electric field model of transcranial electric stimulation in nonhuman primates: correspondence to individual motor threshold. Ieee. Trans. Biomed. Eng. 62, 2095-2105 (2015).

78. Datta, A. et al. Gyri-precise head model of transcranial direct current stimulation: improved spatial focality using a ring electrode versus conventional rectangular pad. Brain Stimul. 2, 201-207-207 (2009).

79. Goncalves, S., de Munck, J. C., Verbunt, J. P., Heethaar, R. M. \& da Silva, F. H. In vivo measurement of the brain and skull resistivities using an EIT-based method and the combined analysis of SEF/SEP data. Ieee. Trans. Biomed. Eng. 50, 1124-1128 (2003).

80. Haueisen, J., Ramon, C., Eiselt, M., Brauer, H. \& Nowak, H. Influence of tissue resistivities on neuromagnetic fields and electric potentials studied with a finite element model of the head. Ieee. Trans. Biomed. Eng. 44, 727-735 (1997).

81. Rush, S. \& Driscoll, D. A. Current distribution in the brain from surface electrodes. Anesth. Analg. 47, 717-723 (1968).

82. Logothetis, N. K., Kayser, C. \& Oeltermann, A. In vivo measurement of cortical impedance spectrum in monkeys: implications for signal propagation. Neuron 55, 809-823 (2007).

83. Opitz, A., Falchier, A., Linn, G. S., Milham, M. P. \& Schroeder, C. E. Limitations of ex vivo measurements for in vivo neuroscience. Proc. Natl Acad. Sci. USA 114, 5243-5246 (2017).

84. Chhatbar, P. Y. et al. Evidence of transcranial direct current stimulationgenerated electric fields at subthalamic level in human brain in vivo. Brain Stimul. 11, 727-733 (2018).

85. Buzsáki, G. Rhythms of the Brain (Oxford University Press, Oxford, 2006).

86. Antal, A. \& Paulus, W. Transcranial alternating current stimulation (tACS). Front. Hum. Neurosci. 7, 317 (2013).

87. Helfrich, R. F. et al. Entrainment of brain oscillations by transcranial alternating current stimulation. Curr. Biol. 24, 333-339 (2014).

88. Neuling, T., Rach, S. \& Herrmann, C. S. Orchestrating neuronal networks: sustained after-effects of transcranial alternating current stimulation depend upon brain states. Front. Hum. Neurosci. 7, 161 (2013).

89. Reato, D. et al. Transcranial electrical stimulation accelerates human sleep homeostasis. PLoS. Comput. Biol. 9, e1002898 (2013).

90. Voss, U. et al. Induction of self awareness in dreams through frontal low current stimulation of gamma activity. Nat. Neurosci. 17, 810-812 (2014)

91. Pogosyan, A., Gaynor, L. D., Eusebio, A. \& Brown, P. Boosting cortical activity at Beta-band frequencies slows movement in humans. Curr. Biol. 19, 1637-1641 (2009).

92. Marshall, L., Kirov, R., Brade, J., Molle, M. \& Born, J. Transcranial electrica currents to probe EEG brain rhythms and memory consolidation during sleep in humans. PLoS ONE 6, el6905 (2011).

93. Marshall, L., Helgadottir, H., Molle, M. \& Born, J. Boosting slow oscillations during sleep potentiates memory. Nature 444, 610-613 (2006).

94. Noury, N., Hipp, J. F. \& Siegel, M. Physiological processes non-linearly affect electrophysiological recordings during transcranial electric stimulation. Neuroimage 140, 99-109 (2016).

95. Weinrich, C. A. et al. Modulation of long-range connectivity patterns via frequency-specific stimulation of human cortex. Curr. Biol. 27, 3061-3068 e3063 (2017).

96. Dehaene, S., Changeux, J. P., Naccache, L., Sackur, J. \& Sergent, C. Conscious preconscious, and subliminal processing: a testable taxonomy. Trends Cogn. Sci. 10, 204-211 (2006)

97. Williams, P. E., Mechler, F., Gordon, J., Shapley, R. \& Hawken, M. J. Entrainment to video displays in primary visual cortex of macaque and humans. J. Neurosci. 24, 8278-8288 (2004).

98. Datta, A., Bikson, M. \& Fregni, F. Transcranial direct current stimulation in patients with skull defects and skull plates: high-resolution computational FEM study of factors altering cortical current flow. Neuroimage 52, 1268-1278 (2010).

99. Poreisz, C., Boros, K., Antal, A. \& Paulus, W. Safety aspects of transcranial direct current stimulation concerning healthy subjects and patients. Brain Res. Bull. 72, 208-214 (2007).

100. Dmochowski, J. P., Datta, A., Bikson, M., Su, Y. \& Parra, L. C. Optimized multi-electrode stimulation increases focality and intensity at target. J. Neural Eng. 8, 046011 (2011).

101. Guleyupoglu, B., Schestatsky, P., Edwards, D., Fregni, F. \& Bikson, M. Classification of methods in transcranial electrical stimulation (tES) and evolving strategy from historical approaches to contemporary innovations. $J$. Neurosci. Methods 219, 297-311 (2013).

102. Goats, G. C. Interferential current therapy. Br. J. Sports Med. 24, 87-92 (1990)

103. Fregni, F. et al. A sham-controlled, phase II trial of transcranial direct curren stimulation for the treatment of central pain in traumatic spinal cord injury. Pain 122, 197-209 (2006). 
104. Lefebvre, S. et al. Dual-tDCS enhances online motor skill learning and longterm retention in chronic stroke patients. Front. Hum. Neurosci. 6, 343 (2012).

105. Dehaene, S. \& Changeux, J. P. Experimental and theoretical approaches to conscious processing. Neuron 70, 200-227 (2011).

106. Dagan, M. et al. Multitarget transcranial direct current stimulation for freezing of gait in Parkinson's disease. Mov. Disord. 33, 642-646 (2018).

107. Fritsch, B. et al. Direct current stimulation promotes BDNF-dependent synaptic plasticity: potential implications for motor learning. Neuron 66, 198-204 (2010).

108. Johnstone, A., Levenstein, J. M., Hinson, E. L. \& Stagg, C. J. Neurochemical changes underpinning the development of adjunct therapies in recovery after stroke: A role for GABA? J. Cereb. Blood Flow Metab. 38, 1564-1583 (2017).

109. Monai, $\mathrm{H}$. et al. Calcium imaging reveals glial involvement in transcranial direct current stimulation-induced plasticity in mouse brain. Nat. Commun. 7, 11100 (2016).

110. Ruohonen, J. \& Karhu, J. tDCS possibly stimulates glial cells. Clin. Neurophysiol. 123, 2006-2009 (2012).

111. Walleczek, J. Electromagnetic field effects on cells of the immune system: the role of calcium signaling. FASEB J. 6, 3177-3185 (1992).

112. Huang, Y. J., Samorajski, J., Kreimer, R. \& Searson, P. C. The influence of electric field and confinement on cell motility. PLoS ONE 8, e59447 (2013).

113. Johansson, O. Disturbance of the immune system by electromagnetic fields-A potentially underlying cause for cellular damage and tissue repair reduction which could lead to disease and impairment. Pathophysiology 16, 157-177 (2009).

114. Krook-Magnuson, E., Gelinas, J. N., Soltesz, I. \& Buzsaki, G. Neuroelectronics and biooptics: closed-loop technologies in neurological disorders. JAMA Neurol. 72, 823-829 (2015)

115. Datta, A., Truong, D., Minhas, P., Parra, L. C. \& Bikson, M. Inter-Individual variation during transcranial direct current stimulation and normalization of dose using MRI-derived computational models. Front Psychiatry. 3, 91 (2012).

116. Opitz, A., Paulus, W., Will, S., Antunes, A. \& Thielscher, A. Determinants of the electric field during transcranial direct current stimulation. Neuroimage 109, 140-150 (2015).

117. Truong, D. Q., Magerowski, G., Blackburn, G. L., Bikson, M. \& AlonsoAlonso, M. Computational modeling of transcranial direct current stimulation (tDCS) in obesity: Impact of head fat and dose guidelines. Neuroimage Clin. 2, 759-766 (2013).

118. Huang, Y., Parra, L. C. \& Haufe, S. The New York Head-A precise standardized volume conductor model for EEG source localization and tES targeting. Neuroimage 140, 150-162 (2016).

119. Huang, Y., Datta A., Bikson, M. \& Parra, L. Realistic volumetric-approach to simulate transcranial electric stimulation - ROAST - a fully automated opensource pipeline". Preprint available at https://doi.org/10.1101/217331 (2017).

120. Miranda, P. C., Lomarev, M. \& Hallett, M. Modeling the current distribution during transcranial direct current stimulation. Clin. Neurophysiol. 117, 1623-1629 (2006).

121. Miranda, P. C., Mekonnen, A., Salvador, R. \& Ruffini, G. The electric field in the cortex during transcranial current stimulation. Neuroimage 70, 48-58 (2013).

122. Sadleir, R. J., Vannorsdall, T. D., Schretlen, D. J. \& Gordon, B. Transcranial direct current stimulation (tDCS) in a realistic head model. Neuroimage 51, 1310-1318 (2010).

123. Nielsen, J. D. et al. Automatic skull segmentation from MR images for realistic volume conductor models of the head: Assessment of the state-of-the-art. Neuroimage 174, 587-598 (2018).

124. Ruffini, G., Fox, M. D., Ripolles, O., Miranda, P. C. \& Pascual-Leone, A. Optimization of multifocal transcranial current stimulation for weighted cortical pattern targeting from realistic modeling of electric fields. Neuroimage 89, 216-225 (2014).

125. Noury, N. \& Siegel, M. Phase properties of transcranial electrical stimulation artifacts in electrophysiological recordings. Neuroimage 158, 406-416 (2017).

126. Gebodh, N. et al. Physiologic Artifacts When Combining EEG and tDCS. Brain Stimul. 10, e33 (2017).
127. Fertonani, A., Ferrari, C. \& Miniussi, C. What do you feel if I apply transcranial electric stimulation? Safety, sensations and secondary induced effects. Clin. Neurophysiol. 126, 2181-2188 (2015).

128. Horvath, J. C., Forte, J. D. \& Carter, O. Evidence that transcranial direct current stimulation (tDCS) generates little-to-no reliable neurophysiologic effect beyond MEP amplitude modulation in healthy human subjects: A systematic review. Neuropsychologia 66, 213-236 (2015).

129. Silberstein, S. D. et al. Safety and efficacy of peripheral nerve stimulation of the occipital nerves for the management of chronic migraine: results from a randomized, multicenter, double-blinded, controlled study. Cephalalgia 32, $1165-1179$ (2012).

130. Tsubokawa, T., Katayama, Y., Yamamoto, T., Hirayama, T. \& Koyama, S. Chronic motor cortex stimulation in patients with thalamic pain. J. Neurosurg. 78, 393-401 (1993).

\section{Acknowledgements}

We thank NYU Finding a Cure Against Epilepsy and Seizures (FACES) for sponsoring the Minisymposium Current state of Transcranial Electrical Stimulation: Progress \& Challenges, on December 18, 2017 where the discussions contained in this manuscript arose. In addition, A.L., S.H., O.D., and G.B. receive support from R01 MH 107396.

\section{Author contributions}

A.L. and G.B. have composed and written the review with contributions from M.V., G.K., S.H., M.K., Y.H., A.O., A.M., C.P., B.K., A.B., L.P., L.M. and O.D.

\section{Additional information}

Supplementary Information accompanies this paper at https://doi.org/10.1038/s41467018-07233-7.

Competing interests: A.B. is the founder and owner of Amplipex and Neunos LLCs, which manufacture biosignal amplifiers and stimulator devices. A.B. and G.B. have filed a patent application about the ISP method. A.O. is an inventor on patents and patent applications describing methods and devices for noninvasive brain stimulation. B.K. is a paid consultant for Aqeel, LLC, which develops transcranial stimulation technology. L.P. has shares in Soterix Medical Inc., which develops transcranial stimulation technology. The remaining authors declare no competing interests.

Reprints and permission information is available online at http://npg.nature.com/ reprintsandpermissions/

Publisher's note: Springer Nature remains neutral with regard to jurisdictional claims in published maps and institutional affiliations.

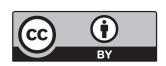

Open Access This article is licensed under a Creative Commons Attribution 4.0 International License, which permits use, sharing, adaptation, distribution and reproduction in any medium or format, as long as you give appropriate credit to the original author(s) and the source, provide a link to the Creative Commons license, and indicate if changes were made. The images or other third party material in this article are included in the article's Creative Commons license, unless indicated otherwise in a credit line to the material. If material is not included in the article's Creative Commons license and your intended use is not permitted by statutory regulation or exceeds the permitted use, you will need to obtain permission directly from the copyright holder. To view a copy of this license, visit http://creativecommons.org/ licenses/by/4.0/.

(c) The Author(s) 2018 